Marek Balcerzak, Institute of Mathematics, Łódź Technical University, al. Politechniki 11, I-2, 90-924 Łódź, Poland and Faculty of Mathematics, University of Łódź, ul. Banacha 22, 90-238 Łódź, Poland. e-mail: mbalce@krysia.uni.lodz.pl Elżbieta Kotlicka, Institute of Mathematics, Łódź Technical University, al. Politechniki 11, I-2, 90-924 Łódź, Poland. e-mail: ekot@ck-sg.p.lodz.pl

\title{
DIFFERENCE PROPERTIES FOR SOME CLASSES OF FUNCTIONS
}

\begin{abstract}
We show the difference property and the double difference property for some classes of real-valued functions.
\end{abstract}

\section{Introduction}

The paper is continuation of $[\mathrm{Ko}]$ and is strictly connected with some results and methods presented in the papers by Laczkovich [L1], [L2], [L3] and Keleti $[\mathrm{Ke}]$.

Let $\mathbb{G}$ stand for the additive group equal to $\mathbb{R}$ or $\mathbb{T}$ where $\mathbb{T}$ is the circle group $\mathbb{R} / \mathbb{Z}$ (and $\mathbb{Z}$ denotes the additive group of all integers). Functions defined on $\mathbb{T}$ can be treated as functions defined on $\mathbb{R}$ and being periodic with period 1. For a fixed function $f: \mathbb{G} \rightarrow \mathbb{R}$ and any $h \in \mathbb{G}$, the difference function $\Delta_{h} f: \mathbb{G} \rightarrow \mathbb{R}$ is defined by

$$
\Delta_{h} f(x)=f(x+h)-f(x),
$$

and the double difference function $D f: \mathbb{G}^{2} \rightarrow \mathbb{R}$ is defined by

$$
D f(x, y)=f(x+y)-f(x)-f(y) .
$$

Let $\mathcal{F}$ and $\mathcal{F}^{(2)}$ be fixed families of functions from $\mathbb{G}$ to $\mathbb{R}$ and from $\mathbb{G}^{2}$ to $\mathbb{R}$, respectively. We say that $\mathcal{F}$ (respectively, the pair $\left(\mathcal{F}, \mathcal{F}^{(2)}\right)$ ) possesses the difference property (respectively, the double difference property), if every function $f: \mathbb{G} \rightarrow \mathbb{R}$ such that $\Delta_{h} f \in \mathcal{F}$ (respectively, $D f \in \mathcal{F}^{(2)}$ ) for each

Key Words: Difference property, double difference property, ideal of sets

Mathematical Reviews subject classification: 26A21, 26A15, 39A70, 03E50

Received by the editors July 11, 2000 
$h \in \mathbb{G}$, is of the form $f=g+A$ where $g \in \mathcal{F}$ and $A$ is an additive function. A class $\mathcal{F}$ is called translation invariant if, for any $f \in \mathcal{F}$ and $a, b \in \mathbb{G}$, the function $g(x):=f(x+a)+b, x \in \mathbb{G}$, belongs to $\mathcal{F}$. If $A \subset \mathbb{G}$ and $x \in \mathbb{G}$, we write $A+x=\{a+x: a \in A\}$.

We consider ideals of subsets of $\mathbb{G}\left(\right.$ or $\mathbb{G}^{2}$ ). Throughout the paper, we assume that every ideal $\mathcal{I}$ has the following properties:

- $\{x\} \in \mathcal{I}$ for each $x \in \mathbb{G}$,

- each set in $\mathcal{I}$ has empty interior,

- $\mathcal{I}$ is translation invariant, i.e. $A+x \in \mathcal{I}$ for any $A \in \mathcal{I}$ and $x \in \mathbb{G}$.

If $\mathcal{I}$ is an ideal of subsets of $\mathbb{G}$, we say that a property holds $\mathcal{I}$-almost everywhere on $\mathbb{G}$, or for $\mathcal{I}$-almost all $x \in \mathbb{G}$, if it holds for all points $x \in \mathbb{G}$ except for some of them which form a set in $\mathcal{I}$. A pair $\left(\mathcal{F}, \mathcal{F}^{(2)}\right)$ is called hereditary (respectively, $\mathcal{I}$-hereditary) if all (respectively, $\mathcal{I}$-almost all) sections $f^{y}$ are in $\mathcal{F}$ for every $f \in \mathcal{F}^{(2)}$ (where $f^{y}(x)=f(x, y), x \in \mathbb{G}$ ). (Note that the present definition of a hereditary pair is different from that given in [Ko].) We shall consider the following ideals of subsets of $\mathbb{G}$ :

- $\mathcal{N}=$ the family of Lebesgue null sets,

- $\mathcal{M}=$ the family of meager sets,

- $\mathcal{M}_{0}=$ the family of nowhere dense sets,

- $\mathcal{I}_{0}=$ the family of countable sets.

Let $\mathcal{N}^{(2)}$ stand for the $\sigma$-ideal of Lebesgue null sets in $\mathbb{G}^{2}$. The symbols $\mathcal{M}^{(2)}$, $\mathcal{M}_{0}^{(2)}$ and $\mathcal{I}_{0}^{(2)}$ have the analogous meanings. If $\mathcal{I}$ is an ideal, we denote

$$
\mathcal{I}^{\star}=\left\{A:\left(\exists B \in \mathcal{I} \text {, of type } F_{\sigma}\right) A \subset B\right\} .
$$

Then $\mathcal{I}^{\star}$ forms an ideal contained in $\mathcal{I}$.

\section{$1 \mathcal{I}$-essentially continuous functions and Sierpiński sets}

Assuming CH Sierpiński $[\mathrm{S}]$ constructed a set $E \subset \mathbb{R}$ such that $E \notin \mathcal{N}$, $\mathbb{R} \backslash E \notin \mathcal{N}$ and $(E+h) \backslash E \in \mathcal{N}$ for each $h \in \mathbb{R}$. Erdős (see [dB1, p. 195]) observed that the characteristic function $\chi_{E}$ of $E$ witnesses the lack of the difference property for the family $L_{0}$ of all Lebesgue measurable functions on $\mathbb{R}$. Laczkovich [L3] proved that the nonexistence of a Sierpiński set is equivalent to the difference property for $L_{0}$. He studied the following condition for an invariant ideal $\mathcal{I}$ of sets in an Abelian group $X$ : 
there exists a set $E \subset X$ such that $E \notin \mathcal{I}, X \backslash E \notin \mathcal{I}$ and $(E+h) \backslash E \in \mathcal{I}$ for every $h \in X$.

In our paper this condition will be used for $X=\mathbb{R}$ and it will be denoted by $\left(S_{\mathcal{I}}\right)$.

Let $C$ stand for the space of all continuous functions from $\mathbb{R}$ to $\mathbb{R}$. For a fixed ideal $\mathcal{I}$ of subsets of $\mathbb{R}$ we denote

$$
C_{\mathcal{I}}=\left\{f \in \mathbb{R}^{\mathbb{R}}:(\exists g \in C)\{x: f(x) \neq g(x)\} \in \mathcal{I}\right\} .
$$

Functions in $C_{\mathcal{I}}$ will be called $\mathcal{I}$-essentially continuous. We are going to prove that if $\mathcal{I} \subset \mathcal{N}$ then $\neg\left(S_{\mathcal{I}}\right)$ is equivalent to the difference property for $C_{\mathcal{I}}$.

We need some auxiliary facts.

Proposition 1.1. [Ke, Thm 2.9] If $f \in L_{0}$ and $\Delta_{h} f \in C_{\mathcal{N}}$ for each $h \in \mathbb{R}$, then $f \in C_{\mathcal{N}}$.

Proposition 1.2. Let $\mathcal{I}, \mathcal{J}$ be ideals of subsets of $\mathbb{R}$ with $\mathcal{I} \subset \mathcal{J}$, and let $\mathcal{A}$ be a $\sigma$-algebra of sets such that $\mathcal{I} \subset \mathcal{A}$. The following assertions hold:

(a) If $f \in C_{\mathcal{I}}$ and $\{x: f(x) \neq g(x)\} \in \mathcal{J}$ for some $g \in C$, then $\{x: f(x) \neq$ $g(x)\} \in \mathcal{I}$.

(b) Assume $\neg\left(S_{\mathcal{I}}\right)$. If $f \in C_{\mathcal{J}}$ and $\Delta_{h} f \in C_{\mathcal{I}}$ for each $h \in \mathbb{R}$ then $f \in C_{\mathcal{I}}$.

(c) Assume $\neg\left(S_{\mathcal{I}}\right)$. If $f: \mathbb{R} \rightarrow \mathbb{R}$ and $\left\{x: \Delta_{h} f(x) \neq 0\right\} \in \mathcal{I}$ for each $h \in \mathbb{R}$ then $f$ is $\mathcal{A}$-measurable.

Proof. (a) This is a simple application of the fact that two continuous functions coinciding on a dense set are equal.

(b) Let $f \in C_{\mathcal{J}}$ and $\Delta_{h} f \in C_{\mathcal{I}}$ for each $h \in \mathbb{R}$. Since $f \in C_{\mathcal{J}}$ there is a $g \in C$ such that $Z:=\{x: f(x) \neq g(x)\} \in \mathcal{J}$. If we put $p=f-g$, then $Z=\{x: p(x) \neq 0\}$. In the case $Z \in \mathcal{I}$ we have $f \in C_{\mathcal{I}}$, so assume that $Z \notin \mathcal{I}$. Since $Z \in \mathcal{J}$, we get $\mathbb{R} \backslash Z \notin \mathcal{I}$. Hence by $\neg\left(S_{\mathcal{I}}\right)$ we have $\left(Z-h_{0}\right) \backslash Z \notin \mathcal{I}$ for some $h_{0} \in \mathbb{R}$. On the other hand, $\left(Z-h_{0}\right) \backslash Z \subset\left\{x: \Delta_{h_{0}} p(x) \neq 0\right\}$, so, to obtain a contradiction, let us prove that the last set is in $\mathcal{I}$. By the definition of $Z$ we get $\left\{x: \Delta_{h_{0}} p(x) \neq 0\right\} \subset Z \cup\left(Z-h_{0}\right) \in \mathcal{J}$. Moreover $\Delta_{h_{0}} p=\Delta_{h_{0}} f-\Delta_{h_{0}} g \in C_{\mathcal{I}}$, so from (a) it follows that $\left\{x: \Delta_{h_{0}} p(x) \neq 0\right\} \in \mathcal{I}$.

(c) (See [L3, Thm 7].) Suppose that $f$ is not $\mathcal{A}$-measurable. Thus there is a $c \in \mathbb{R}$ with $E:=\{x: f(x)>c\} \notin \mathcal{A}$. From $\mathcal{I} \subset \mathcal{A}$ it follows that $E \notin \mathcal{I}$ and $\mathbb{R} \backslash E \notin \mathcal{I}$. Let $h \in \mathbb{R}$. Observe that $(E-h) \backslash E \subset\left\{x: \Delta_{h} f(x) \neq 0\right\} \in \mathcal{I}$. Hence $\left(S_{\mathcal{I}}\right)$ holds true, contrary to our assumption.

Theorem 1.3. Let $\mathcal{I}$ be an ideal of sets in $\mathbb{R}$ such that $\mathcal{I} \subset \mathcal{N}$. Then the condition $\neg\left(S_{\mathcal{I}}\right)$ is equivalent to the difference property for $C_{\mathcal{I}}$. 
Proof. (I) The demonstration that $\left(S_{\mathcal{I}}\right)$ excludes the difference property for $C_{\mathcal{I}}$ goes back to the idea of Erdős. Namely, if $\left(S_{\mathcal{I}}\right)$ holds true, pick an $E \notin \mathcal{I}$ with $\mathbb{R} \backslash E \notin \mathcal{I}$ and $(E+h) \backslash E \in \mathcal{I}$ for each $h \in \mathbb{R}$. Hence for $f=\chi_{E}$ we have $f \notin C_{\mathcal{I}}$ and $\Delta_{h} f \in C_{\mathcal{I}}$ for each $h \in \mathbb{R}$. Suppose that $C_{\mathcal{I}}$ has the difference property. Then $f=g+A$ where $g \in C_{\mathcal{I}}$ and $A$ is additive. Hence $A=f-g$ is additive and bounded on a set of positive measure, so (by Ostrowski's theorem [Os] $) A$ is continuous and consequently, $f \in C_{\mathcal{I}}$.

(II) Now assume $\neg\left(S_{\mathcal{I}}\right)$. Let $f: \mathbb{R} \rightarrow \mathbb{R}$ be such that $\Delta_{h} f \in C_{\mathcal{I}}$ for each $h \in \mathbb{R}$. By [Ke, Thm 2.13] the function $f$ admits a decomposition $f=g+A+\varphi$ where $g \in C_{\mathcal{N}}, A$ is additive and $Z_{h}:=\left\{x: \Delta_{h} \varphi(x) \neq 0\right\} \in \mathcal{N}$ for each $h \in \mathbb{R}$. Since $g \in C_{\mathcal{N}}$, there exists an $r \in C$ with $B:=\{x: g(x) \neq r(x)\} \in \mathcal{N}$. Let $p=g-r$. Then $p \in C_{\mathcal{N}}$ and $B=\{x: p(x) \neq 0\}$. Observe that $\Delta_{h} p(x)=0$ for any $x, h \in \mathbb{R}$ with $x \notin B \cup(B-h)$. Consequently,

$$
(\forall h, x \in \mathbb{R})\left(x \notin B \cup(B-h) \cup Z_{h} \Rightarrow \Delta_{h}(p+\varphi)(x)=0\right) .
$$

On the other hand, $p+\varphi=g-r+\varphi=f-A-r$. Hence $\Delta_{h}(p+\varphi)=$ $\Delta_{h} f-A(h)-\Delta_{h} r$ for each $h \in \mathbb{R}$. Thus from the assumption that $\Delta_{h} f \in C_{\mathcal{I}}$ for each $h \in \mathbb{R}$, and from the continuity of $r$, it follows that $\Delta_{h}(p+\varphi) \in C_{\mathcal{I}}$ for each $h \in \mathbb{R}$. This together with (1) and Proposition 1.2(a) implies that $\{x$ : $\left.\Delta_{h}(p+\varphi)(x) \neq 0\right\} \in \mathcal{I}$. Now, from Proposition 1.2(c) we infer that $p+\varphi \in L_{0}$. Consequently $\varphi \in L_{0}$. Since $\Delta_{h} \varphi \in C_{\mathcal{N}}$ for each $h \in \mathbb{R}$, by Proposition 1.1 we get $\varphi \in C_{\mathcal{N}}$. Now $g+\varphi \in C_{\mathcal{N}}$ and $\Delta_{h}(g+\varphi)=\Delta_{h}(f-A)=\Delta_{h} f-A(h)$ is in $C_{\mathcal{I}}$ for each $h \in \mathbb{R}$, which by Proposition 1.2(b) means that $g+\varphi \in C_{\mathcal{I}}$.

By the theorem of Trzeciakiewicz $[\mathrm{T}]$, we have $\left(S_{\mathcal{I}_{0}}\right) \Longleftrightarrow \mathrm{CH}$. (See also [L3, Remark 2, p.668].) Thus we obtain

Corollary 1.4. $\neg \mathrm{CH}$ is equivalent to the difference property for $C_{\mathcal{I}_{0}}$.

Remarks. 1. For $\mathcal{I}=\mathcal{N}$, the condition $\left(S_{\mathcal{I}}\right)$ is independent of ZFC [L3]. Hence, by Theorem 1.3, the difference property for $C_{\mathcal{N}}$ is independent of ZFC. We expect similar results for $\mathcal{I}=\mathcal{N}^{\star}$, and for $\mathcal{I}$ equal to the $\sigma$-ideal of $\sigma$-porous sets. To have this, one needs models of ZFC in which $\neg\left(S_{\mathcal{I}}\right)$ is false. From [L3, Thm 2] it follows that $\neg\left(S_{\mathcal{I}}\right)$ is implied by $\operatorname{cov}(\mathcal{I})>\operatorname{non}^{\star}(\mathcal{I})$, thus it suffices to find models in which $\operatorname{cov}(\mathcal{I})>\operatorname{non}^{\star}(\mathcal{I})$ holds. Note that models with $\operatorname{cov}(\mathcal{I})>\operatorname{non}(\mathcal{I})$ for $\mathcal{I}=\mathcal{N}^{\star}$ and $\mathcal{I}=\sigma$-porous sets were found in [BJ, 2.6] and [R, Thms 1 and 6], respectively. However, this is not enuogh since unfortunately $\operatorname{non}(\mathcal{I}) \leq \operatorname{non}^{\star}(\mathcal{I})[\mathrm{L} 3$, Thm 2]. For the definitions of $\operatorname{cov}(\mathcal{I})$, $\operatorname{non}(\mathcal{I})$ and $\operatorname{non}^{\star}(\mathcal{I})$, see [L3].

2. Let us consider $\mathcal{M}$ instead of $\mathcal{N}$ in Theorem 1.3. Part (I) of the proof still works since we can use Mehdi's theorem $[\mathrm{M}]$ instead of Ostrowski's theorem. Part (II) works provided any $f$, with $\Delta_{h} f \in C_{\mathcal{M}}$ for each $h \in \mathbb{R}$, 
admits a decomposition $f=g+A+\varphi$ where $g \in C_{\mathcal{M}}, A$ is additive and $\left\{x: \Delta_{h} \varphi(x) \neq 0\right\} \in \mathcal{M}$. However, we do not know whether the last property holds true.

3. Assume $\mathrm{CH}$. Observe that, if $\mathcal{I}$ is a $\sigma$-ideal such that $\mathcal{I} \subset \mathcal{N}$ or $\mathcal{I} \subset \mathcal{M}$, then $C_{\mathcal{I}}$ does not have the difference property. It suffices to use the Erdoss type argument based on the Sierpiński set [S] and its category analog. In fact, the proofs for $\mathcal{I}=\mathcal{N}$ and $\mathcal{I}=\mathcal{M}$ are contained in [Ke, Thm 2.11] and [BKW, Thm 2.2]. A general case is similar.

\section{Some Classes of Functions With the Difference Prop- erty}

A real-valued function on $\mathbb{R}$ is called pointwise discontinuous if its set of continuity points is dense or, equivalently, its set of discontinuity points is meager. Laczkovich in [L2] proved that the family of pointwise discontinuous functions on $\mathbb{R}$ has the difference property. From the following lemma we shall derive that some important subclasses of this family also possess the difference property.

Lemma 2.1. Let $\mathcal{F}$ be equal to the family of all pointwise discontinuous functions on $\mathbb{R}$ and let $\mathcal{G}$ be a subfamily of $\mathcal{F}$ invariant under addition of constants. If

$$
\forall f \in \mathcal{F}\left(\left(\forall h \in \mathbb{R} \quad \Delta_{h} f \in \mathcal{G}\right) \Rightarrow f \in \mathcal{G}\right)
$$

then $\mathcal{G}$ has the difference property.

Proof. (Cf. [Ke, Lemma 1.1]). Assume that $f: \mathbb{R} \rightarrow \mathbb{R}$ and $\Delta_{h} f \in \mathcal{G}$ for each $h \in \mathbb{R}$. Since $\mathcal{G} \subset \mathcal{F}$ and $\mathcal{F}$ has the difference property, we have $f=g+A$ where $g \in \mathcal{F}$ and $A$ is additive. Thus $\Delta_{h} f=\Delta_{h} g+A(h)$ which implies that $\Delta_{h} g \in \mathcal{G}$ (for each $h \in \mathbb{R}$ ). Hence, by $(2)$, we get $g \in \mathcal{G}$.

First consider the family of functions continuous $\mathcal{I}$-almost everywhere where $\mathcal{I}$ is a given ideal. Since the set of discontinuity points of any function is of type $F_{\sigma}$, the functions continuous $\mathcal{I}$-almost everywhere coincide with those continuous $\mathcal{I}^{\star}$-almost everywhere. Since (by our preliminary claim) each set in $\mathcal{I}$ has empty interior, we have $\mathcal{I}^{\star} \subset \mathcal{M}$. Thus it follows that each function continuous $\mathcal{I}$-almost everywhere is continuous $\mathcal{M}$-almost everywhere or, in other words, it is pointwise discontinuous.

Lemma 2.2. For $f: \mathbb{R} \rightarrow \mathbb{R}$ let $\omega(f, x)$ denote the oscillation of $f$ at a point $x \in \mathbb{R}$. For an arbitrary $h \in \mathbb{R}$, if $\Delta_{h} f$ is continuous at a point $x_{0}$ then $\Delta_{h} \omega\left(f, x_{0}\right)=0$. 
Proof. We have $\omega(f, x)=\bar{f}(x)-f(x)$ where $\bar{f}(x)=\max \left\{f(x), \limsup _{t \rightarrow x} f(t)\right\}$, $\underline{f}(x)=\min \left\{f(x), \liminf _{t \rightarrow x} f(t)\right\}$ for $x \in \mathbb{R}$. Since $f(x+h)=\Delta_{h} f(x)+f(x)$ for each $x \in \mathbb{R}$ and $\lim _{x \rightarrow x_{0}} \Delta_{h} f(x)=\Delta_{h} f\left(x_{0}\right)$, we have $\bar{f}\left(x_{0}+h\right)=$ $\Delta_{h} f\left(x_{0}\right)+\bar{f}\left(x_{0}\right)$ and $f\left(x_{0}+h\right)=\Delta_{h} f\left(x_{0}\right)+f\left(x_{0}\right)$. Hence $\omega\left(f, x_{0}+h\right)=$ $\bar{f}\left(x_{0}+h\right)-\underline{f}\left(x_{0}+h\right)=\bar{f}\left(x_{0}\right)-\underline{f}\left(x_{0}\right)$ and thus $\Delta_{h} \omega\left(f, x_{0}\right)=0$.

Theorem 2.3. Let $\mathcal{I}$ be an ideal of subsets of $\mathbb{R}$. If $\neg\left(S_{\mathcal{I}^{\star}}\right)$ then the family of all functions continuous $\mathcal{I}$-almost everywhere on $\mathbb{R}$ has the difference property.

Proof. We shall use Lemma 2.1 with $\mathcal{G}$ equal to the family of functions continuous $\mathcal{I}$-almost everywhere. So, we shall check condition (2). Let $f$ be pointwise discontinuous, and let $\Delta_{h} f$ be continuous $\mathcal{I}$-almost everywhere, for each $h \in \mathbb{R}$. Fix an $h \in \mathbb{R}$. By Lemma 2.2 we have $\Delta_{h} \omega(f, x)=0$ at each point $x$ of continuity of $\Delta_{h} f$. Consequently, $\Delta_{h} \omega(f, \cdot)$ is equal $\mathcal{I}^{\star}$-almost everywhere to a continuous (zero) function. On the other hand, $\{x \in \mathbb{R}: \omega(f, x) \neq 0\} \in \mathcal{M}$ since $f$ is pointwise discontinuous. From $\neg\left(S_{\mathcal{I}^{\star}}\right)$, inclusion $\mathcal{I}^{\star} \subset \mathcal{M}$ and Proposition 1.2(b) it follows that $\omega(f, \cdot) \in C_{\mathcal{I}^{\star}}$. Moreover, by Proposition $1.2\left(\right.$ a) we have $\{x \in \mathbb{R}: \omega(f, x) \neq 0\} \in \mathcal{I}^{\star}$ which means that $f$ is continuous $\mathcal{I}$-almost everywhere.

Corollary 2.4. The family of all functions continuous $\mathcal{M}_{0}$-almost everywhere on $\mathbb{R}$ has the difference property.

Proof. We use the fact that $\neg\left(S_{\mathcal{M}_{0}}\right)$ is true. (See [L2, Remark 7].)

We shall show that, in some cases, the assumption $\neg\left(S_{\mathcal{I}^{*}}\right)$ in Theorem 2.3 is superfluous.

Theorem 2.5. The family of all functions continuous $\mathcal{I}_{0}$-almost everywhere on $\mathbb{R}$ has the difference property.

PRoof. In a former version of the paper, the above statement was derived under $\neg \mathrm{CH}$ from Theorem 2.3 and the equivalence $\left(S_{\mathcal{I}_{0}}\right) \Longleftrightarrow \mathrm{CH}$. Recently, I. Recław has communicated us the following ZFC proof. Apply Lemma 2.1 with $\mathcal{G}$ equal to the set of all functions continuous $\mathcal{I}_{0}$-almost everywhere. We need to check condition (2), so suppose it is false. Thus there is an $f \in \mathcal{F}$ such that $\Delta_{h} f \in \mathcal{G}$ for each $h \in \mathbb{R}$ and the set $F$ of discontinuity points of $f$ is uncountable. Pick a perfect set $P \subset F$ and a countable set $D \subset P$ dense in $P$. Since $F$ is meager, there is an $h \in \mathbb{R}$ such that $(F-h) \cap D=\emptyset$. Then $(\mathbb{R} \backslash(F-h)) \cap P$ is uncountable (as a dense $G_{\delta}$ set in $P$ ) and $f(x+h)-f(x)$ is discontinuous at each point of $(\mathbb{R} \backslash(F-h)) \cap P$ because $f(x+h)$ is continuous at each point of this set and $f(x)$ is discontinuous. Contradiction.

Theorem 2.6. The family of all functions continuous $\mathcal{N}$-almost everywhere on $\mathbb{R}$ has the difference property. 
Proof. We check condition (2) of Lemma 2.1 where $\mathcal{G}$ stands for the set of functions continuous $\mathcal{N}$-almost everywhere. Let $f$ be pointwise discontinuous, and let $\Delta_{h} f$ be continuous $\mathcal{N}$-almost everywhere for each $h \in \mathbb{R}$. As in the proof of Theorem 2.3, we infer from Lemma 2.2 that $\Delta_{h} \omega(f, \cdot) \in C_{\mathcal{N}}$ for each $h \in \mathbb{R}$. Since $\omega(f, \cdot)$ is upper-semicontinuous (hence measurable), therefore by Proposition 1.1, it belongs to $C_{\mathcal{N}}$. Thus there is a continuous function $g$ such that $\{x \in \mathbb{R}: \omega(f, x)=g(x)\}$ is of full measure. This set is dense of type $G_{\delta}$, so it is comeager. On the other hand, $\{x \in \mathbb{R}: \omega(f, x)=0\}$ is comeager since $f$ is pointwise discontinuous. It implies that $g=0$ everywhere which means that $f$ is continuous $\mathcal{N}$-almost everywhere.

Remark. It is well known that the functions continuous $\mathcal{N}$-almost everywhere bounded on a given compact interval are exactly the Riemann integrable functions. Note that the difference property for the family of Riemann integrable functions was shown by de Bruijn [dB2]. His method of proof is different. We do not know how to derive Theorem 2.6 from de Bruijn's result.

In [L1, Thm 8] it is proved that the family of all approximately continuous functions on $\mathbb{R}$ has the difference property. A category analog of approximately continuous functions was introduced in [PWW], and those functions will be called category approximately continuous. We are going to give two different proofs of the difference property for category approximately continuous functions.

Proposition 2.7. Let $H \subset \mathbb{R}$ and $H \notin \mathcal{M}$ (respectively, $H \notin \mathcal{N}$ ). If a function $f: \mathbb{R} \rightarrow \mathbb{R}$ has the Baire property (is measurable) and $\Delta_{h} f$ is category approximately continuous (approximately continuous) for every $h \in H$ then so is $f$.

Proof. Let $B$ stand for the set of category approximate continuity (respectively, the approximate continuity) points of $f$. It is known that $B$ is comeager (respectively, of full measure). (See e.g. [CLO, Thms 1.3.2, 2.5.6].) Thus for any $x_{0} \in \mathbb{R}$ we have $B \cap\left(x_{0}+H\right) \neq \emptyset$ and so, there exists an $h_{0} \in H$ such that $x_{0}+h_{0} \in B$. From $f(x)=f\left(x+h_{0}\right)-\Delta_{h_{0}} f(x)$ and from the assumptions it follows that $x_{0} \in B$.

Theorem 2.8. The family of all category approximately continuous functions on $\mathbb{R}$ has the difference property.

Proof. (I) Consider the statement of Lemma 2.1 with $\mathcal{G}$ equal to the set of all category approximately continuous functions (they are in Baire class 1 and consequently, they are pointwise discontinuous). Obviously pointwise 
discontinuous functions have the Baire property. Thus Proposition 2.7 yields the condition (2) in Lemma 2.1.

(II) We give a category analogue of the argument in [L1, Thm 8]. Since $\Delta_{h} f$ is category approximately continuous for every $h$, therefore the function $D f$ is separately category approximately continuous and by [BLW] it is of Baire class 2. Then by [L1, Thm 7] we have $f=g+A$ where $g$ is of Baire 2 and $A$ is additive. Pick a point $x_{0}$ at which $g$ is category approximately continuous (the set of such points is comeager). Then $g(x+h)=g(x)+\Delta_{h} f(x)-A(x)$ implies that $g$ is category approximately continuous at $x_{0}+h$. Since $h$ is arbitrary, $g$ is category approximately continuous everywhere.

Remark. The first of the above arguments can be used in the measure case, too. Namely, the Baire class 1 has the difference property (that has been derived by Laczkovich [L4] from his main result of [L2]). We use this class as $\mathcal{F}$ in the statement of Lemma 2.1. The role of $\mathcal{G}$ is played by the approximately continuous functions. (Obviously such a version of Lemma 2.1 works with the same proof.)

Recall $[\mathrm{Km}]$ that a function $f: \mathbb{R} \rightarrow \mathbb{R}$ is said to be quasi-continuous at a point $x_{0} \in \mathbb{R}$ if, for any open neighbourhoods $U$ of $x_{0}$, and $V$ of $f\left(x_{0}\right)$, there exists a nonempty open set $G \subset U$ such that $f[G] \subset V$. A function is called quasi-continuous on $\mathbb{R}$ if it is quasi-continuous at each point of $\mathbb{R}$. Quasi-continuous functions of two variables are defined analogously.

Theorem 2.9. The family of all quasi-continuous functions on $\mathbb{R}$ has the difference property.

Proof. It is known that every quasi-continuous function is pointwise discontinuous. To get the assertion we use Lemma 2.1. In fact, we shall prove that, for an $H \subset \mathbb{R}$ with $H \notin \mathcal{M}$, if a function $f: \mathbb{R} \rightarrow \mathbb{R}$ is pointwise discontinuous and $\Delta_{h} f$ is quasi-continuous for each $h \in H$ then $f$ is quasi-continuous. Thus, let $H$ and $f$ be as above. Denote by $E$ the set of continuity points of $f$. Then $E$ is comeager. Let $x_{0} \in \mathbb{R}$. There exists an $h \in H$ such that $x_{0}+h \in E$. Hence $f(x)=f(x+h)-\Delta_{h} f(x)$ is quasi-continuous at $x_{0}$ as a sum of a function continuous at $x_{0}$ and a function quasi-continuous at $x_{0}$.

\section{Some Classes of Functions With the Double Difference Property}

From the definitions given in Introduction we immediately derive the following lemma 
Lemma 3.1. Let $\mathcal{F}$ and $\mathcal{F}^{(2)}$ be fixed families of functions from $\mathbb{G}$ to $\mathbb{R}$ and from $\mathbb{G}^{2}$ to $\mathbb{R}$, respectively. If $\left(\mathcal{F}, \mathcal{F}^{(2)}\right)$ is hereditary and $\mathcal{F}$ has the difference property, then $\left(\mathcal{F}, \mathcal{F}^{(2)}\right)$ has the double difference property.

Another variant of this lemma was proved in [Ko, Prop.1]:

Lemma 3.2. Let $\mathcal{F}$ and $\mathcal{F}^{(2)}$ be fixed families of functions from $\mathbb{G}$ to $\mathbb{R}$ and from $\mathbb{G}^{2}$ to $\mathbb{R}$, respectively. Assume that $\mathcal{F}$ constitutes a translation invariant additive group and $\mathcal{I}$ is a $\sigma$-ideal of subsets of $\mathbb{G}$. If $\left(\mathcal{F}, \mathcal{F}^{(2)}\right)$ is $\mathcal{I}$-hereditary and $\mathcal{F}$ has the difference property, then $\left(\mathcal{F}, \mathcal{F}^{(2)}\right)$ has the double difference property.

Remark. Lemma 3.2 remains true if $\mathcal{I}$ is an ideal (not necessarily a $\sigma$-ideal) since the same proof given in [Ko] works.

Theorem 3.3. Let $\mathcal{J} \in\left\{\mathcal{M}, \mathcal{N}, \mathcal{M}_{0}, \mathcal{I}_{0}\right\}$. If $\mathcal{F}$ (respectively, $\left.\mathcal{F}^{(2)}\right)$ stands for the family of all functions from $\mathbb{G}$ (respectively, $\mathbb{G}^{2}$ ) to $\mathbb{R}$ that are continuous $\mathcal{J}$-almost (respectively, $\mathcal{J}^{(2)}$-almost) everywhere, then $\left(\mathcal{F}, \mathcal{F}^{(2)}\right)$ has the double difference property.

Proof. For $\mathcal{J}=\mathcal{M}$, the theorem was proved in [Ko, Thm 1]. For $\mathcal{J}=\mathcal{N}$, we use Theorem 2.6, Lemma 3.2 and the Fubini theorem. For $\mathcal{J}=\mathcal{M}_{0}$, observe that the respective pair $\left(\mathcal{F}, \mathcal{F}^{(2)}\right)$ is $\mathcal{M}$-hereditary, by a version of the Kuratowski-Ulam theorem [O, Thm 15.1]. So, Corollary 2.4 and Lemma 3.2 yield the assertion. Similarly, for $\mathcal{J}=\mathcal{I}_{0}$ we use Theorem 2.5 and Lemma 3.2 .

In the sequel, the family of category approximately continuous functions on $\mathbb{R}$ will be denoted by $C A C$. Similarly as in the measure case (see [GNN]), there are two standard variants of the notion of a category density point for plane sets. They were introduced and described in $[\mathrm{CW}]$ and [BLW]. We call them an ordinary category density point and a strong category density point. The both notions generate, in a usual way, topologies that are named the ordinary category density topology and the strong category density topology in the plane. (See $[\mathrm{CW}]$. .) In turn, if we consider any of these topologies in the domain, and the natural topology on $\mathbb{R}$ - in the range, the respective continuous functions from $\mathbb{R}^{2}$ to $\mathbb{R}$ are called ordinarily category approximately continuous and strongly category approximately continuous functions of two variables. The family of these last functions will be denoted by $S C A C$. From [BLW, Thm 1.4] it follows that the pair $(C A C, S C A C)$ is hereditary.

The following notion of a function from $[0,1]^{2}$ to $\mathbb{R}$ with finite variation was introduced by Idczak in [I]. Namely, $f:[0,1]^{2} \rightarrow \mathbb{R}$ is said to be of finite variation if the functions $f(\cdot, 0), f(0, \cdot)$ are of finite variation and the associated 
interval function $F_{f}$ defined by

$$
F_{f}(P)=f(\bar{x}, \bar{y})-f(\bar{x}, y)-f(x, \bar{y})+f(x, y)
$$

for $P=[x, \bar{x}] \times[y, \bar{y}] \subset[0,1]^{2}$, has a finite variation. It was observed in $[\mathrm{I}]$ that, for every function $f:[0,1]^{2} \rightarrow \mathbb{R}$ of finite variation and for any $x, y \in[0,1]$, the functions $f(x, \cdot), f(\cdot, y)$ are of finite variation. The above notions and properties can easily be adapted to the case when $f: \mathbb{T}^{2} \rightarrow \mathbb{R}$. Thus, if $B V\left(\mathbb{T}^{2}\right)$ (respectively, $B V(\mathbb{T})$ ) denotes the family of real-valued functions with finite variation on $\mathbb{T}^{2}$ (respectively, on $\mathbb{T}$ ) then the pair $\left(B V(\mathbb{T}), B V\left(\mathbb{T}^{2}\right)\right.$ ) is hereditary.

By Lemma 3.1, from the above facts, Theorem 2.8 and the result of de Bruijn [dB1] that $B V(\mathbb{T})$ has the difference property, we obtain:

Theorem 3.4. The pairs $(C A C, S C A C)$ and $\left(B V(\mathbb{T}), B V\left(\mathbb{T}^{2}\right)\right)$ have the double difference property.

Remark. Let $Q C$ and $Q C^{(2)}$ denote the families of all quasi-continuous functions on $\mathbb{R}$ and $\mathbb{R}^{2}$, respectively. The newest result of $[\mathrm{KoM}]$ states that the pair $\left(Q C, Q C^{(2)}\right)$ is $\mathcal{M}$-hereditary. Hence from Lemma 3.1 and Theorem 2.9 it follows that this pair has the double difference property.

In the sequel we shall use the following result from [Ko, Thm 2]:

Lemma 3.5. Let $\mathcal{F}$ and $\mathcal{F}^{(2)}$ be families of functions from $\mathbb{G}$ to $\mathbb{R}$ and from $\mathbb{G}^{2}$ to $\mathbb{R}$, respectively. Assume that $\mathcal{F}$ is an additive group of functions such that every additive function from $\mathcal{F}$ is linear. Let $\mathcal{G}$ be a subgroup of $\mathcal{F}$ containing all linear functions and let $\mathcal{G}^{(2)} \subset \mathcal{F}^{(2)}$. If $\left(\mathcal{F}, \mathcal{F}^{(2)}\right)$ has the double difference property, then the following conditions are equivalent:

(a) $\forall f \in \mathcal{F}\left(D f \in \mathcal{G}^{(2)} \Rightarrow f \in \mathcal{G}\right)$,

(b) $\left(\mathcal{G}, \mathcal{G}^{(2)}\right)$ has the double difference property.

Let $C\left(\mathbb{G}^{i}\right), U C\left(\mathbb{G}^{i}\right)$ and $\operatorname{Lip}\left(\mathbb{G}^{i}\right)$ denote, respectively, the families of continuous, uniformly continuous and Lipschitz functions from $\mathbb{G}^{i}$ to $\mathbb{R}$ (where $i=1,2)$. It is known that for $\mathbb{G}=\mathbb{T}$ the classes $U C(\mathbb{G})(=C(\mathbb{G}))$ and $\operatorname{Lip}(\mathbb{G})$ have the difference property. (See $[\mathrm{dB} 1],[\mathrm{BBL}]$ and $[\mathrm{Ke}]$.) In the case $\mathbb{G}=\mathbb{R}$, the analogs of these results are false which can be easily shown by the use of the function $f(x)=x^{2}, x \in \mathbb{R}$. We are going to prove that the pairs $\left(U C(\mathbb{R}), U C\left(\mathbb{R}^{2}\right)\right)$ and $\left(\operatorname{Lip}(\mathbb{R}), \operatorname{Lip}\left(\mathbb{R}^{2}\right)\right)$ have the double difference property. Our method of proof is based on Lemma 3.5.

Proposition 3.6. Assume that an $f: \mathbb{R} \rightarrow \mathbb{R}$ is continuous at 0 . If $D f \in$ $U C\left(\mathbb{R}^{2}\right)$ then $f \in U C(\mathbb{R})$. 
Proof. Let $\varepsilon>0$. There exist $\delta_{1}, \delta_{2}>0$ such that $|f(x)-f(0)|<\varepsilon / 2$ for each $x \in \mathbb{R}$ with $|x|<\delta_{1}$, and $|D f(p)-D f(q)|<\varepsilon / 2$ for any $p, q \in \mathbb{R}^{2}$ with the Euclidean norm $\|p-q\|<\delta_{2}$. Put $\delta=\min \left\{\delta_{1}, \delta_{2} / \sqrt{2}\right\}$. Then, for any $x, y \in \mathbb{R}$ with $|x-y|<\delta$, we obtain $|f(x)-f(y)|=\mid D f(x-y, y)-D f(0, x)+$ $f(x-y)-f(0)|\leq| D f(x-y, y)-D f(0, x)|+| f(x-y)-f(0) \mid<\varepsilon$.

Proposition 3.7. If $f \in U C(\mathbb{R})$ and $D f \in \operatorname{Lip}\left(\mathbb{R}^{2}\right)$ then $f \in \operatorname{Lip}(\mathbb{R})$.

Proof. The idea of the proof comes from [BBL, Thm 2, proof of (ii) $\Rightarrow(\mathrm{i})]$. By assumption there exist $L, \delta>0$ such that $|D f(x, h)-D f(y, h)| \leq L|x-y|$ for any $x, y, h \in \mathbb{R}$, and $|f(x)-f(y)| \leq 1$ whenever $|x-y|<\delta$. Fix an $h_{0} \in(0, \delta)$. Thus $\left|\Delta_{h_{0}} f(x)\right| \leq 1$ for each $x \in \mathbb{R}$. Let $x, y \in \mathbb{R}$. Consider the integral

$$
I_{x y}=\int_{0}^{h_{0}}(f(y+h)-f(x+h)) d h .
$$

One can easily check that we have $I_{x y}=\int_{x}^{y} \Delta_{h_{0}} f(h) d h$. Thus $\left|I_{x y}\right| \leq|x-y|$. Now we have $|f(x)-f(y)|=\left|\left(1 / h_{0}\right) \int_{0}^{h_{0}}(f(x)-f(y)) d h\right|=\mid\left(1 / h_{0}\right) \int_{0}^{h_{0}}(D f(x, h)-$ $D f(y, h)+f(y+h)-f(x+h)) d h\left|\leq\left(1 / h_{0}\right) \int_{0}^{h_{0}}\right| D f(x, h)-D f(y, h) \mid d h+$ $\left(1 / h_{0}\right)\left|I_{x y}\right|<L|x-y|+\left(1 / h_{0}\right)|x-y|=\left(L+\left(1 / h_{0}\right)\right)|x-y|$.

Theorem 3.8. The pairs $\left(U C(\mathbb{R}), U C\left(\mathbb{R}^{2}\right)\right)$ and $\left(\operatorname{Lip}(\mathbb{R}), \operatorname{Lip}\left(\mathbb{R}^{2}\right)\right)$ have the double difference property.

Proof. We know that the pair $\left(C(\mathbb{R}), C\left(\mathbb{R}^{2}\right)\right)$ has the double difference property, by [dB1] and Lemma 3.1. Proposition 3.6 shows that condition (a) in Lemma 3.5 is true with $\mathcal{F}=C(\mathbb{R}), \mathcal{F}^{(2)}=C\left(\mathbb{R}^{2}\right), \mathcal{G}=U C(\mathbb{R})$ and $\mathcal{G}^{(2)}=U C\left(\mathbb{R}^{2}\right)$. So, condition (b) of Lemma 3.5 yields the first assertion of our theorem. Similarly we deduce the second assertion from Proposition 3.7 and from the first assertion.

Acknowledgements. We would like to thank the referee for several remarks that have improved our paper. We also thank Irek Recław who has permitted us to include his ZFC proof of Theorem 2.5 to this article.

\section{References}

[BBL] M. Balcerzak, Z. Buczolich, M. Laczkovich, Lipschitz differences and Lipschitz functions, Colloq. Math. 157 (1998), 15-32.

[BKW] M. Balcerzak, E. Kotlicka, W. Wojdowski, Difference functions for functions with the Baire property, Aequationes Math. 57 (1999), 278287. 
[BLW] M. Balcerzak, E. Łazarow, W. Wilczyński, On one- and twodimensional $\mathcal{I}$-densities and related kinds of continuity, Real Anal. Exchange 13 (1987-88), 80-92, 120-121, 130-150.

[BJ] T. Bartoszyński, H. Judah, Set Theory: On the Structure of the Real Line, A K Peters, Wellesley, Massachusets, 1995.

[dB1] N.G. de Bruijn, Functions whose differences belong to a given class, Nieuw Arch. Wisk. 23 (1951), 194-218.

[dB2] N.G. de Bruijn, A difference property for Riemann integrable functions and for similar classes of functions, Indag. Math. 14 (1952), 145-151.

[CW] R. Carrese, W. Wilczyński, I-density points of plane sets, Ricerche Mat. 34 (1985), 147-157.

[CLO] K. Ciesielski, L. Larson, K. Ostaszewski, I-density continuous functions, Mem. Amer. Math. Soc. 107, no 515 (1994).

[GNN] C. Goffman, C. J. Neugebauer, T. Nishiura, Density topology and approximate continuity, Duke Math. J. 28 (1961), 497-506.

[I] D. Idczak, Functions of several variables of finite variation and their differentiability, Ann. Polon. Math. 60 (1994), 47-56.

[Ke] T. Keleti, Difference functions of periodic measurable functions, Fund. Math. 157 (1998), 15-32.

[Km] S. Kempisty, Sur les fonctions quasicontinues, Fund. Math. 19 (1932), 184-197.

[Ko] E. Kotlicka, The double difference property for some classes of functions, Real Anal. Exchange 25 (1999-2000), 463-468.

$[\mathrm{KoM}]$ E. Kotlicka, A. Maliszewski, On quasi-continuity and cliquishness of sections for functions of two variables, submitted.

[L1] M. Laczkovich, Functions with measurable differences, Acta Math. Hungar. 35 (1980), 217-235.

[L2] M. Laczkovich, On the difference property of the class of pointwise discontinuous functions and some related classes, Canad. J. Math. 36 (1984), 756-768.

[L3] M. Laczkovich, Two constructions of Sierpiński and some cardinal invariants of ideals, Real Anal. Exchange 24(2) (1998/9), 663-676. 
[L4] M. Laczkovich, Difference property of various classes of functions, Summer School on Real and Spectral Analysis, Wrocław, 1999 (unpublished talk).

[M] M. R. Mehdi, On convex functions, J. London Math. Soc. 39 (1964), $321-326$.

[Os] A. Ostrowski, Über die Funktionalgleichung der Exponentialfunktion und verwandte Funktionalgleichungen, Jahresber. Deutschen Math. Verein., 38 (1929), 54-62.

[O] J.C. Oxtoby, Measure and Category, Springer, New York, 1971.

[PWW] W. Poreda, E. Wagner-Bojakowska, W. Wilczyński, A category analogue of the density topology, Fund. Math. 125 (1985), 167-173.

[R] M. Repický, Cardinal invariants related to porous sets, in: Set Theory of the Reals (H. Judah, ed.), Israel Math. Conf. Proc. 6 (1993), 433-438.

[S] W. Sierpiński, Sur les translations des ensembles linéaires, Fund. Math. 19 (1932), 22-28.

[T] L. Trzeciakiewicz, Remarque sur les translations des ensembles linéaires, C.R. Soc. Lettres Varsovie C1.III.25 (1933), 63-65. 
